\title{
Male smokers see the light; females left puffing in the dark
}

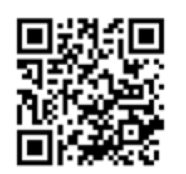

Tobacco smoking causes lung carcinoma in $90 \%$ of cases in developed countries. ${ }^{[1]}$ Since the introduction of the antismoking laws in South Africa in 1993, there has been a dramatic reduction in cigarette smoking in men. In women, the number of smokers is much lower but unfortunately has not shown any reduction. Using the data from the South Africa Demographic and Health Survey in 2003, which provides detailed smoking history regarding age, sex and ethnicity, combined with longitudinal estimates of smoking prevalence from 1980 to 2010, Winkler et al.${ }^{[1]}$ predicted a decrease in adjusted lung cancer mortality in South Africa from 17.1 to 14.1 ASA (age-standardised mortality rates) per 100000 among men over the period 2010 - 2025. Rates are stable for women. They also predicted the highest mortality rates for Asian men and lowest for black men. In women, the lowest rates were in Asian women and the highest in white and mixed ancestry women.

In this issue, the paper by Pellizzon et al. ${ }^{[2]}$ provides information on the effect of smoking on the histological cell type of lung cancer in the Western Cape, and in particular the drainage area of Tygerberg Hospital, using 386 participants. They used immunohisto- and immunocytochemistry to assist in cell typing of the lung cancer. As they noted, Asian and black patients were underrepresented, so that their results cannot be applied elsewhere in South Africa.

Traditionally, squamous cell carcinoma was the most frequent cell type of lung cancer presenting as a central lung mass, like small cell carcinoma, and adenocarcinoma and large cell carcinoma were peripheral in distribution. Main bronchi should have the highest exposure to inhaled carcinogens in cigarette smoke whereas there would be less effect in the peripheral regions of the lung. Squamous carcinomas frequently undergo central necrosis and cavitation, which in a South African setting might provide diagnostic radiological confusion with tuberculosis and a delay in diagnosis. Adenocarcinoma was second in frequency.

Currently, the distinction between central and peripheral squamous tumours has become less well-defined, with equal numbers of squamous carcinomas arising peripherally and centrally. In many parts of the world, in particular the USA and Japan, adenocarcinomas have become the most frequent type of lung carcinoma. Cigarettes have undergone changes in manufacturing over the years with lowtar filters becoming more popular, and with this type of cigarettes the carcinogens now penetrate more deeply into the lungs, explaining in part the current change in distribution. Previously, cigarette smoking was a predominantly male custom, whereas currently many more women are smoking. Customs of smoking are also different in different ethnic groups.

Histological typing of lung cancer has always been problematic because of tumour heterogeneity, with the most accurate typing possible in surgically resected specimens. The current trend is towards small endoscopic biopsies, fine-needle aspiration and sputum cytology, all of which provide small samples of the cancer. Therefore, the histological typing of lung carcinomas will vary according to the source of the material. Biopsy and cytology usually have a predominance of cases being squamous carcinomas followed by adenocarcinomas. A surgical series will consist predominantly of resectable peripheral tumours. A hospital-based postmortem series will reflect rapidly disseminated lung cancers. A large community-based survey for lung carcinomas over a 10 -year period should be the most reliable. However, smoking patterns may change with time and cause a difference in histologic type. When mortality from lung cancer in workers with asbestosis is used, adenocarcinoma is the most frequent cell type.

The World Health Organization (WHO)'s criteria for diagnosing lung cancer has also changed over the last decade, with the use of immunostains and mucin stains enabling more adenocarcinomas to be diagnosed. Histological typing has become of paramount importance because of advances in cancer therapy requiring molecular testing in adenocarcinomas. Precursor lesions for adenocarcinomas have been described; previously precursor lesions were only known for squamous carcinomas. The WHO has created new entities in the adenocarcinoma group, such as solid adenocarcinoma with mucin production, resulting in a marked drop in the diagnosis of large cell carcinoma. If one includes electron microscopy (EM) as an adjunct in cell typing there would be no large cell carcinomas. Using EM adenosquamous carcinoma becomes the most common association of smoking in central tumours.

In addition, there is a genuine increase in adenocarcinoma in non-smokers and young women of Asian ethnicity. There may be geographic variations in the prevalence of adenocarcinoma: in Japan $3 \%$ arose centrally, whereas $17 \%$ occurred centrally in the UK. The other important aspect of adenocarcinomas is its high propensity to metastasise, so preoperative screening of the brain is important.

Pellizzon et al. found an increase in adenocarcinomas in nonsmokers. ${ }^{[2]}$ It would be interesting to determine whether this finding is present in other academic centres in South Africa.

They found no difference between smokers and non-smokers with scar-associated lung cancer. Patients with fibrosing alveolitis have been shown to have an increased incidence of lung cancer due to fibrosis. The presence of fibrosis associated with and not preceding a lung cancer usually indicates an advanced aggressive cancer with lymphatic and vascular invasion.

Lung cancer is a devastating disease with an overall 5-year survival of $17 \%$ at best, owing to late presentation, early metastases and poor sustained response to chemotherapy or development of resistance. Surgery offers a 5 -year survival of $50 \%$ only with rigorous preoperative assessment excluding $80 \%$ of cases.

Further antismoking campaigns are required. Currently, smokers are permitted to smoke outdoors. Hospitals need to bring in regulations that require smokers to smoke outside the hospital perimeter, making it extremely inconvenient for staff and patients to smoke. This has been implemented in Australia. The high cost of cigarettes has had a beneficial effect in that many women cannot afford more than 2 3 cigarettes a day. The first campaign showed a significant reduction in childhood smoking and this is an ideal age to target. Targeting of specific ethnic groups could also be implemented.

1. Winkler V, Mangolo NJ, Becker H. Lung cancer in South Africa: A forecast to 2025 based on smoking prevalence data. BMJ open 2015 Mar 17;5(3):e006993. [http:// dx.doi.org/10.1136/bmjopen-2014-006993]

2. Pellizzon AS, Koegelenberg CFN, Irusen EM. A 2-year retrospective review of the effect of cigarette smoking status on the histological cell types of lung carcinoma in the Western Cape. S Afr Resp J 2015; 21(2):23-28. [http://dx.doi.org/10.7196/sarj.8441]

\section{Helen Wainwright}

FFPath (Anat Path); Associate Professor, Department of Pathology, Faculty of Health Sciences, University of Cape Town, South Africa

S Afr Resp J 2015;21(2):22. DOI:10.7196/sarj.8440 